\title{
El tenis en el nivel elite - Un análisis del centro técnico de la ITF
}

\author{
Jamie Capel-Davies
}

Federación Internacional de Tenis

\section{RESUMEN}

Cumpliendo con su misión, el Centro Técnico cuantifica los parámetros que describen la naturaleza actual del tenis a nivel elite, y de este modo, constituye "el estado del juego". El análisis anual del estado del juego que realiza el Centro Técnico consta de un análisis del jugador, e incluye las estadísticas de los partidos, y el monitoreo del equipamiento que utiliza.
Palabras clave: : análisis de jugadores, análisis de equipos, estadísticas de partidos

Recibido: 26 Mayo 2017

Aceptado: 01 Noviembre 2017

Autor correspondiente: Jamie Capel-Davies

Federación Internacional de Tenis.

Correo electrónico: jamie.capeldavies@itftennis.com

\section{ANÁLISIS DEL JUGADOR}

\section{Fisiología}

La Figura 1 muestra que la estatura promedio de las mejores 50 mujeres ha aumentado $1 \mathrm{~cm}$. desde 2002, y $4 \mathrm{~cm}$. en el caso de los mejores 50 hombres. Este incremento de la estatura media de los hombres se debe a la cantidad de jugadores activos que superan los $200 \mathrm{~cm}$ - en 2002 no había ninguno- y a un incremento general de la estatura de todos estos 50 jugadores (Figura 2).

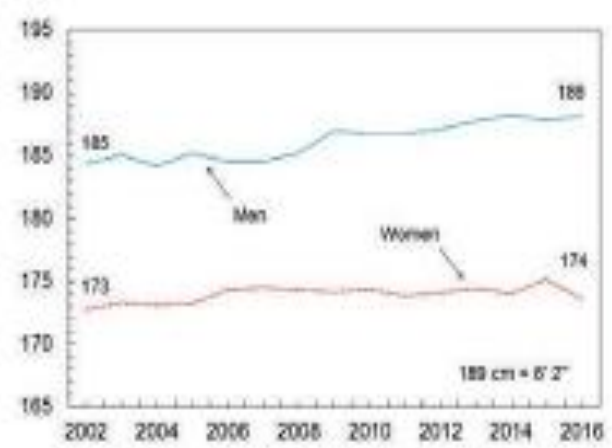

Figuro 1. Estaturo promedio de los mejores 50 hombres (azul) y mujeres (rojo).
Los jugadores más altos suelen tener un mayor alcance con el brazo, le imprimen una mayor velocidad a la cabeza de la raqueta (para una velocidad de movimiento de preparación constante), y por lo tanto, logran un servicio más rápido. Además, los más altos pueden servir con una trayectoria más empinada y un mayor margen de error. En 2002, las mejores 50 mujeres eran, como promedio, $12 \mathrm{~cm}$. más bajas que sus pares masculinos. Esta diferencia se ha incrementado ahora a $15 \mathrm{~cm}$. La mujer más alta entre las mejores 50 en la actualidad, es $4 \mathrm{~cm}$. más baja que el promedio de estatura de los mejores 50 hombres $(189 \mathrm{~cm})$.

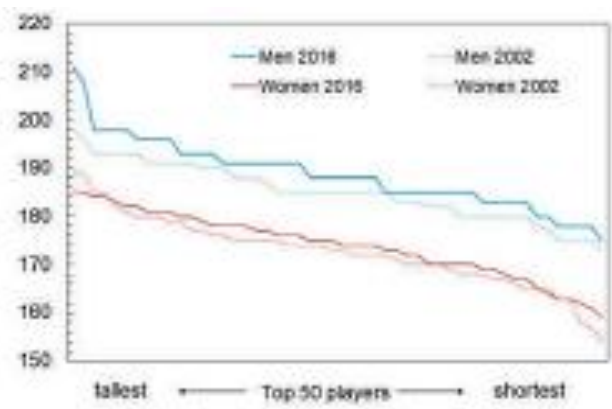

Figura 2. Estatura individual de los mejores so hombres (arull) en 2002 yer 2016 .

La Figura 3 muestra que desde 2002, la edad promedio de las mejores 50 mujeres incrementó dos años, y la de los mejores 
50 hombres, 3 años. En 2002, pocos jugadores entre los mejores 50, hombres y mujeres, superaban los 30 años. Actualmente, casi la mitad de los mejores 50 masculinos están en la tercera década (Figura 4). Esto indica que las carreras de los mejores se están extendiendo, y menos jugadores jóvenes irrumpen entre los mejores 50 .

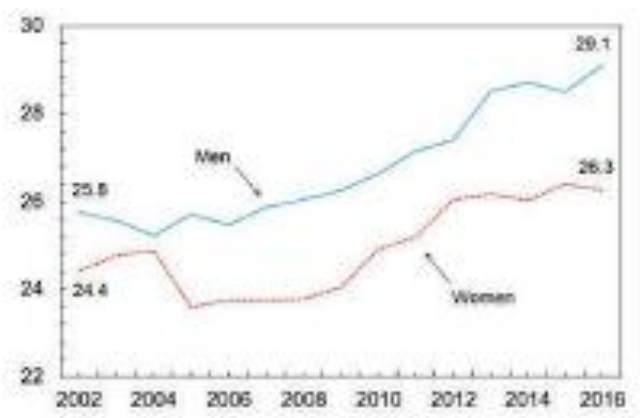

Figura 3- Edad promedio de los mejores 50 varones (arul) $y$ mujeres (rojol.

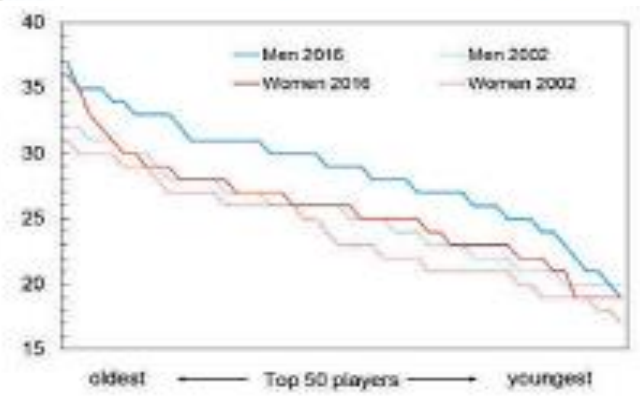

Figura 4- Edades individuales de las mejores 50 varones (ozully $y$ mujeres (nojo) en 2002 y en 2016.

\section{Velocidad y éxito del servicio}

Tanto en el juego masculino como femenino, la velocidad del servicio aumentó entre 2002 y 2005 antes de alcanzar una meseta en la mayoría de los Grand Slams (figura 5). Desde 2012, la velocidad del servicio en el Abierto de Australia está aumentando tanto para hombres como para mujeres. Durante la última década, los 20 sacadores masculinos más rápidos lo hicieron a un promedio de $220 \mathrm{~km}$.h-1 y las mujeres a 185 km.h-1. La única desviación mayor de estos valores durante este período fue en la competición femenina de Roland Garros 2010, con un anormal $195 \mathrm{~km} . \mathrm{h} 1$.

La Figura 6 muestra que la incidencia de los "aces" en el juego femenino se mantiene razonablemente estable desde 2002. En el caso de los hombres, la incidencia de los "aces" en Wimbledon ha incrementado y prácticamente duplica a Roland Garros en gran parte del período. Durante éste, los hombres sirvieron "aces" duplicando la tasa de las mujeres, lo cual puede atribuirse a la diferencia promedio de $35 \mathrm{~km} . \mathrm{h}-1$ en la velocidad del servicio entre los géneros. Las Figuras 5 y 6 sugieren que el incremento de estatura en el juego de los hombres no solamente incide en la velocidad del servicio, sino también en la cantidad de "aces" (ya que la estatura adicional puede ayudar a mejorar la colocación del servicio).

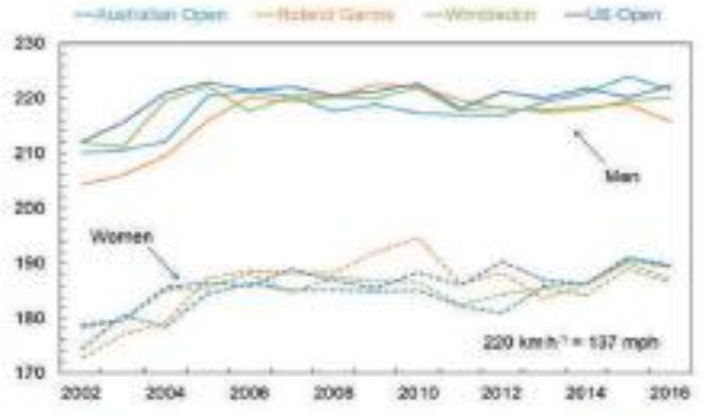

Figura 5. Velbcidad de servicia promedio para los zo servidores más rupidos en los individuales de Grand Slam.

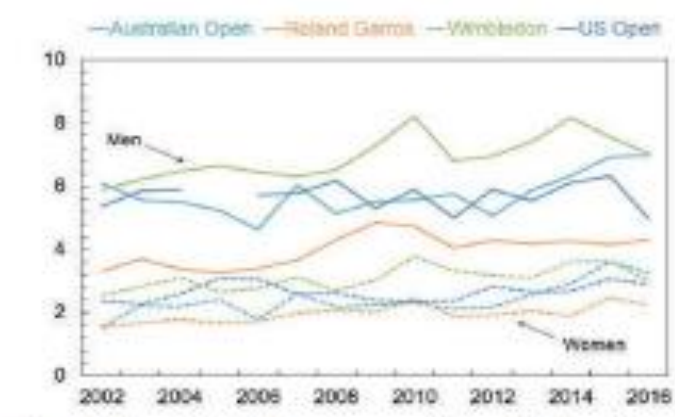

Figuna 6. Porcentaje de "aces" parservicio en indíviduales de Grand Slam.

El servicio fue más potente en Wimbledon, lo cual es un testimonio de la influencia de la velocidad de la cancha (figura 7). El reciente incremento en la velocidad del servicio en el Abierto de Australia se correlaciona con el incremento en la tasa de "aces" y puntos ganados con el servicio. Sorprendentemente, el incremento de unos $10 \mathrm{~km} . \mathrm{h}-1$, en la velocidad del servicio entre 2002 y 2005 tuvo poca incidencia en los puntos ganados con el servicio (o "aces"). Es también notable que a pesar de que los "aces" fueron menos frecuentes en Roland Garros, los puntos ganados con el saque fueron comparables con los del Abierto de Australia y el Abierto de los Estados Unidos entre 2006 y 2013.

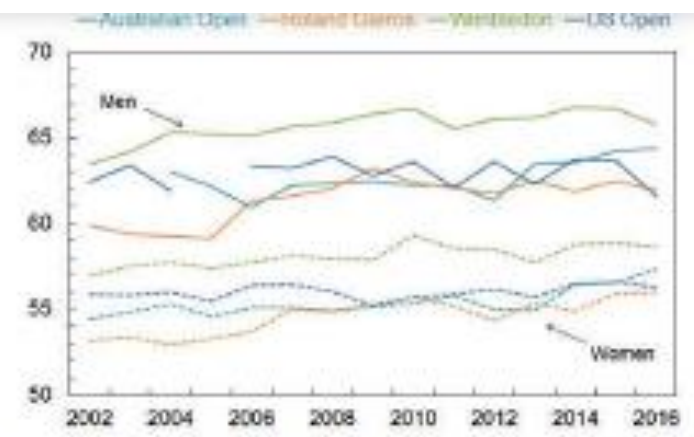

Figura $z$ Porcentaje de puntas ganados con el servicio en las individuaies de Grand Sham. 


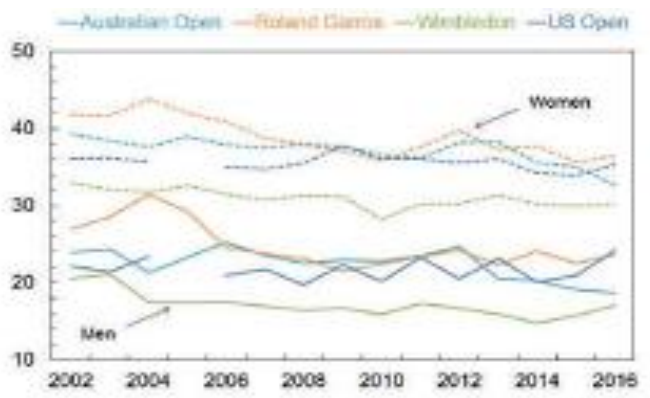

Figura s. Parcentaje de juegos quebrados en los individuales de Grand Slam.

La figura 8 muestra el impacto de los puntos ganados con el servicio en los juegos quebrados. El servicio fue menos dominante en el juego femenino que en el masculino. En los últimos 10 años, el porcentaje promedio de juegos quebrados en los partidos masculinos fue inferior al $25 \%$ en todos los Grand Slams y $20 \%$ en Wimbledon.

\section{ANÁLISIS DEL EQUIPAMIENTO}

\section{Raquetas}

La Figura 9 muestra la variación en la masa de encordado de las raquetas usadas por los mejores 50 hombres y mujeres en 2016.

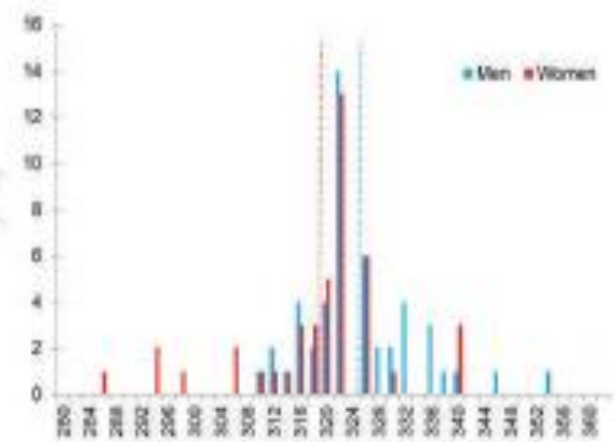

Figunag. Distribución del peso de las raquetas encordadas utilizados por los mejores so hombres (onil) y mujeres (rojo) en 2016 (datos de la Universidad de Tenis Warehouse) L. La lineas punteadas verticales indican los valores medios para cada génera.

El peso de raqueta más común para hombres y mujeres fue de $322 \mathrm{~g}$, pero el promedio para hombres fue $6 \mathrm{~g}$ más (325 g contra $319 \mathrm{~g}$ ).racket mass was $6 \mathrm{~g}$ heavier ( $325 \mathrm{~g}$ compared to $319 \mathrm{~g})$.

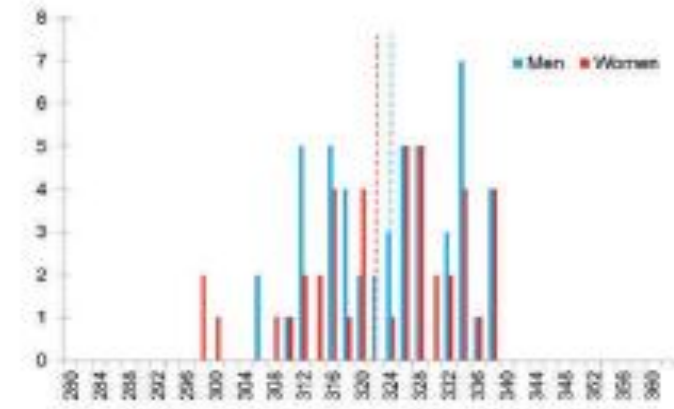

Figura 10. La distribución del swingweight del encordado para las raquetas utilizadas por las 50 mejores hombres (azul) y mujeres (rajo) en 2016 (datos de la Universidad de Tenis Warehouse). Lo lineas punteados verticales indican los valores medios para cada génera.

Hubo una pequeña diferencia en la variación del swingweight (distribución de masa dentro del marco) de las raquetas utilizadas por los mejores 50 varones y mujeres en 2016 (Figura 10). El swingweight promedio para hombres fue de 324 $\mathrm{kg} \cdot \mathrm{cm} 2$, y para mujeres de $322 \mathrm{~kg} \cdot \mathrm{cm} 2$. El mayor peso y swingweight de la raqueta generan mayor velocidad de servicio (para una velocidad de movimiento de preparación dada).

\section{Pelotas}

La Figura 11 muestra una tendencia general hacia una mayor altura del bote de las pelotas, en los torneos hasta 2013, que indicaría mayor velocidad del servicio (todos los otros factores son iguales). Sin embargo, en todos los tres últimos años el promedio de altura del bote de la pelota en torneos ha disminuido. La altura media del bote de la pelota en torneos es ahora la misma que la de las pelotas enviadas para su aprobación (que típicamente se ha mantenido cerca del punto medio de las especificaciones desde 2002).

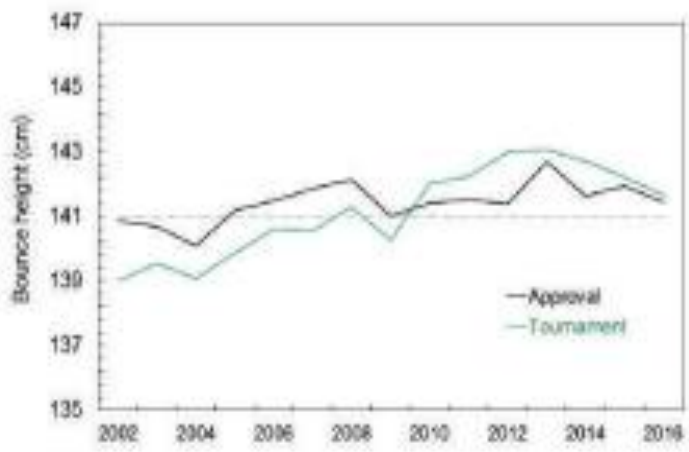

Figura n. La altura media del bate de los pelotos presurizados para torneos y oprobación. La línea punteada indica el punto medio de la especificación. 


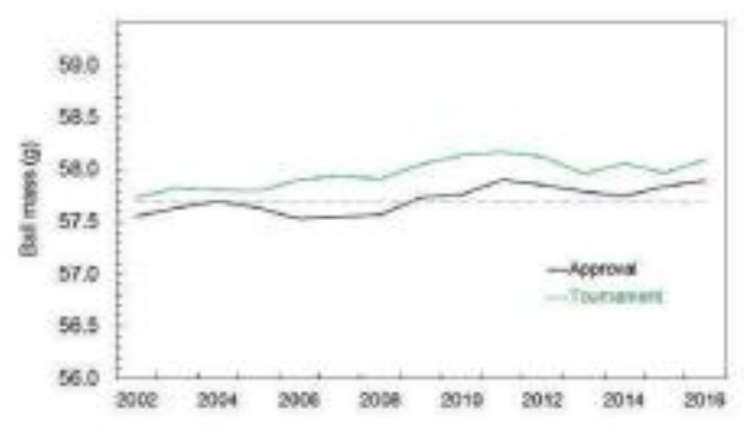

Figura 22. Masa promedio de las pelotos presurizadas para tomeas yaprobación. La Nnea harizontal punteado indica el punto medio de la especificación.

La Figura 12 indica que la masa de las pelotas de torneo ha aumentado levemente con el tiempo, y produce un efecto ínfimo sobre la velocidad del servicio (cuando llega a quien recibe) y es continuamente superior a la de las pelotas enviadas por los fabricantes para su aprobación. Los valores medios para las pelotas de torneo y aprobación se han mantenido dentro de los $0,5 \mathrm{~g}$ del punto medio de las especificaciones $(57.7 \mathrm{~g})$.

\section{CONCLUSIÓN}

La estatura media de los mejores 50 ha aumentado desde 2002: $1 \mathrm{~cm}$, a $174 \mathrm{~cm}$, para las mujeres; y $4 \mathrm{~cm}$, a $189 \mathrm{~cm}$ para los hombres. Estos incrementos de estatura no han tenido una incidencia notable sobre la velocidad del servicio de los 20 mejores y más rápidos sacadores en los Grand Slams. Los hombres sirvieron, como promedio, $35 \mathrm{~km} . \mathrm{h}-1$ más rápido que las mujeres, duplicando la frecuencia de "aces". La efectividad del servicio ha aumentado en Roland Garros, y más recientemente en el Abierto de Australia:, pero aún sigue siendo más potente en Wimbledon. El servicio es un tiro clave en el juego, y constituye más de un cuarto de todos los tiros en Copa Davis y Fed Cup.

Los mejores 50 hombres solían utilizar raquetas ligeramente más pesadas que las mujeres en 2016, y hubo escasa diferencia en el swingweight de las raquetas entre los géneros. Por lo tanto, la elección de la raqueta, improbablemente incida en la velocidad del servicio de hombres y mujeres. La altura media del bote de la pelota usada en torneos ha disminuido en estos tres últimos años, a tal punto, que se acerca al punto medio de la especificación ( $\mathrm{y}$ al valor de las pelotas enviadas para su aprobación). La media de la masa de las pelotas usadas en los torneos ha aumentado ligeramente, pero se mantiene cerca del punto medio de las especificaciones.

\section{REFERENCIAS}

Tennis Warehouse University (2016). Racquet Comparison Tool. Disponible en: http://twu.tennis-warehouse.com/cgi-bin/ racquetspecs2.cgi (Acceso 5 Diciembre 2016).

\section{CONTENIDO ITF ACADEMY RECOMENDADO (HAZ CLICK ABAJO)}

\section{$\widehat{T I F}^{\circ}$ Academy}

Derechos de Autor (c) 2017 Jamie Capel-Davies

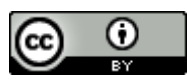

Este texto está protegido por una licencia CreativeCommons $\underline{4.0}$.

Usted es libre para Compartir -copiar y redistribuir el material en cualquier medio o formato- y Adaptar el documento -remezclar, transformar y crear a partir del material- para cualquier propósito, , incluso para fines comerciales, siempre que cumpla la condición de:

Atribución: Usted debe dar crédito a la obra original de manera adecuada, proporcionar un enlace a la licencia, e indicar si se han realizado cambios. Puede hacerlo en cualquier forma razonable, pero no de forma tal que sugiera que tiene el apoyo del licenciante o lo recibe por el uso que hace de la obra.

$\underline{\text { Resumendelicencia - Textocompletodelalicencia }}$ 